\title{
Player Aggregation in Noncooperative Games, II*
}

\author{
A. J. Goldman $\dagger$ \\ The Johns Hopkins University, Baltimore, MD 21218
}

April 1, 1981

\begin{abstract}
Conditions are given under which subsets of the players of a noncooperative game can be combined into "aggregate players" without changing the set of equilibrium-point solutions of the game. These conditions are shown to be the weakest possible ones with a certain specified kind of generality. "Approximate" versions of the results are also formulated and proven.
\end{abstract}

Key words: Aggregation; equilibrium; game theory; mathematical economics; noncooperative games; total orders.

\section{Introduction}

Like its predecessor [1], ${ }^{1}$ this paper is motivated by the following observation: in applied game-theoretic modeling, it may prove useful to reduce model-complexity and data-needs by combining, into a single "aggregate player," some subset of the original players whose interests are sufficiently "parallel" for this purpose. It is natural, therefore, to investigate from a mathematical viewpoint the conditions under which such an aggregation is "valid" in the sense of leaving the game's set of "solutions" undisturbed. Results of this type were needed, for example, in connection with a class of models $[2,3,4]$ involving an inspection agency with insufficient resources to visit all of a number of sites at which "cheating" might or might not occur; the issue was whether the individual site-managers could be aggregated into a single "inspectee" entity so that the situation could be analyzed as a two-person game.

Our treatment here is limited to noncooperative games and to the equilibrium-point notion of "solution." For completeness, these concepts are defined in section 2 below, where the process of aggregation is also formalized. A natural special case of aggregation ("group equilibrium") is discussed in [5], but without considering the question of solution-set preservation.

In [1], a simple condition was given under which aggregation does not change a game's set of solutions. That condition stated that each individual player's payoff (in the original game) is independent of the strategy choices by the other individuals comprising the same aggregate player. Though applicable to the inspector-inspectee games mentioned above, this condition is clearly rather restrictive, and captures the "independence" or "indifference" of the aggregated players' interests rather than (as desired) the "parallelism" of those interests. In section 3 of the present paper, we give less restrictive sufficient conditions for aggregation (a) to avoid introducing extraneous solutions, and (b) to preserve all solutions of the original game. Further theorems and examples show that these conditions, though not necessary as well as sufficient, are actually the weakest possible sufficient conditions with a certain specified kind of generality. Additional examples show that the family of player-subsets which are "aggregable," in the sense of obeying the minimal conditions mentioned above, need not have certain properties to be expected if aggregability fully corresponded to some natural notion of "parallel interest;" for example, a subset of an aggregable set need not be aggregable.

-AMS Subject Classification: 90D10,90D40,90D45, 06A05

†Department of Mathematical Sciences, The Johns Hopkins University, Baltimore, MD 21218, and the Center for Applied Mathematics, National Engineering Laboratory, National Bureau of Standards, Washington, DC 20234.

' Numbers in brackets indicate literature references at the end of the paper. 
Consistent with [1], section 4 extends the preceding material to "approximate solutions" in cases in which the conditions described above are satisfied only approximately. This topic reflects an expectation that in applied contexts, many mathematical relationships will not (or cannot be known to) hold exactly.

I am grateful to colleague D. R. Shier, whose careful reading of a first version of this paper revealed the need for several corrections and clarifications.

\section{Games, equilibria, aggregation}

Let $n \geqslant 1$ be an integer, and $N=\{1,2, \ldots, n\}$. An $n$-person noncooperative game $G=(X, f)$ consists of an $n$-tuple $\left(X_{1}, \ldots, X_{n}\right)$ of nonempty sets $X_{i}$ with Cartesian product $X$, and an $n$-tuple $f=\left(f_{1}, \ldots, f_{n}\right)$ of functions $f_{i}: X \rightarrow R_{i}$ where $R_{i}$ is equipped with a strict linear order $\varrho_{i}$. Here $X_{i}$ is interpreted as the set of strategies or actions open to the $i$-th player, $f_{i}$ as that player's "payoff function," $R_{i}$ as the set of possible payoffs or outcomes experienced by that player, and $\varrho_{i}$ as the relation of (strict) preference by that player among outcomes. The fact that the domain of $f_{i}$ is $X$, rather than $X_{i}$, expresses the idea that each player's payoff depends not only on what strategy that player chooses, but also on the choices made by other players.

For any $x \varepsilon X$, any $i \varepsilon N$, and any $x_{i} \varepsilon X_{i}$, we denote by $\left(x, i, x_{i}\right)$ the member of $X$ obtained from $x$ by changing its $i$-th coordinate to $x_{i}$. With this notation, a "solution" concept can be defined: $x^{\circ} \varepsilon X$ is called an equilibrium point $(E P)$ for game $G$ if, for every $i \varepsilon N$ and every $x_{i} \varepsilon X_{i}$, the relation

$$
f_{i}\left(x^{\circ}, i, x_{i}\right) \varrho f_{i}\left(x^{\circ}\right)
$$

is false. That is, if one thinks of the coordinates of $x^{\circ}$ as the players" "current" choices of strategies, then no player has an incentive to deviate unilaterally from his or her current choice. Since the game is regarded as "noncooperative," only unilateral shifts come into consideration, and so the falsity of all relations (l) is sufficient to describe the "stability" of $x^{\circ}$. If $n=1$, an $E P$ is simply a strategy that yields a preference-maximal outcome for the (sole) player.

Next we describe an "aggregation" of game $G$. Let $m$ be an integer with $1 \leqslant m \leqslant n$, and let $M=$ $\{1,2, \ldots, m\}$. An $m$-player aggregation $G B, F]$ of $G$ is specified by the following structure. $B=\left\{B_{1}, \ldots, B_{m}\right\}$ is a partition of $N$ into nonempty sets; note that the relation $i \varepsilon B_{j(i)}$ defines a function $j: N \rightarrow M$. Let $S_{j}$ be the Cartesian product of the sets $\left\{R_{i}: i \varepsilon B_{j}\right\}$; also let $F=\left(F_{1}, \ldots, F_{m}\right)$ be an $m$-tuple of functions $F_{j}: S_{j} \rightarrow T_{j}$ where each set $T_{j}$ is equipped with a strict linear order $\tau_{j}$, and function $F_{j}$ is strictly monotone in each of its arguments. This last condition means that for any $s_{j} \varepsilon S_{j}$, for any $i \varepsilon B_{j}$ with $r_{i}$ the $i$-th coordinate of $s_{j}$, and for any $r_{i}^{\prime} \varepsilon R_{i}$,

$$
r_{i}^{\prime} \varrho_{i} r_{i} \text { implies } F_{j}\left(s_{j}, i, r_{i}^{\prime}\right) \tau_{j} F_{j}\left(s_{j}\right)
$$

Such a structure defines an $m$-person game as follows. The "players" are $\left\{B_{j}: j \varepsilon M\right\}$. The set of strategies of $B_{j}$ is $Y_{j}$, the Cartesian product of $\left\{X_{i}: i \varepsilon B_{j}\right\}$. Note that the Cartesian product of the player's strategy-sets, i.e. of $\left\{Y_{j}: j \varepsilon M\right\}$, is the same set $X$ as for the original game; this permits the symbols " $x$ " and " $y$ " to be used interchangeably, and corresponds to the idea that we are dealing with aggregation of players and payoffs, but not of strategies. (The same observation justifies the later use of notation like $\left(x, j, y_{j}\right)$, as an extension of the previous symbol $\left(x, i, x_{i}\right)$.) In the aggregated game, the payoff function for player $B_{j}$ is $g_{j}$ : $X \rightarrow T_{j}$, defined by ${ }^{2}$

$$
g_{j}(x)=F_{j}\left[\left\{f_{i}(x): i \varepsilon B_{j}\right\}\right]
$$

The definition of an $E P$ for game $G[B, F]$ is directly analogous to that for $G$.

${ }^{2}$ In the following notation, the argument of $F$, is the member $s_{f} S_{j}$, whose $i$-th coordinate, for $i \in B_{j}$, is $f_{i}(x)$. 


\section{Preservation of the solution-set}

For any subset $S$ of $N$, let $X_{S}$ denote the Cartesian product of the strategy-sets $\left\{X_{i}: i \varepsilon S\right\}$. We will call $x \varepsilon X$ $S$-stable if there is no $x_{s} \varepsilon X_{s}$ such that (with an obvious extension of previous notation)

$$
f_{i}\left(x, S, x_{s}\right) \varrho f_{i}(x) \text { or } f_{i}\left(x, S, x_{S}\right)=f_{i}(x)
$$

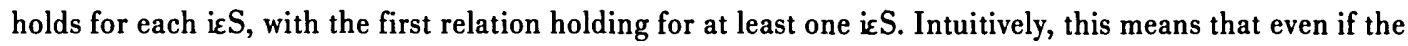
players in $S$ could act together, they could effect no deviation from the status quo represented by $x$ which would be "advantageous to $S$ " in the sense of being preferable to some members of $S$ and a matter of indifference to the others. Thus $x^{\circ}$ is an $E P$ if and only if it is $\{i\}$-stable for all $i \varepsilon N$.

Let $X^{s}$ denote the set of $S$-stable members of $X$. We call $S$ nonfrustrating $(N F)$ if

$$
X^{s} \subseteq \cap\left\{X^{[i\}}: i \varepsilon S\right\}
$$

The reason for this terminology is best seen by taking complements in (4): for each $x \varepsilon X$, if some player iES can find a unilateral deviation preferable (for $\imath$ ) to the status quo represented by $x$, then there is a joint deviation $x_{s \varepsilon} X_{s}$ which is "advantageous to $S$ " in the sense described above and which could therefore be adopted by $\mathrm{S}$ without internal dissension were it the only available alternative to continuation of $x$. That is, there is no opposition of interests within $S$ that would preclude deviating from a status quo which some single player in $S$ could profitably deviate from. Thus no player, by becoming part of an aggregate player $S$, would risk the frustration of being "stuck with" an undesirable status quo against which he or she had a unilateral counteraction as an individual player.

THEOREM 1. If each $\mathrm{B}_{j}$ is $\mathrm{NF}$ and $\mathrm{x}^{\circ}$ is an $\mathrm{EP}$ for $\mathrm{G}[\mathrm{B}, \mathrm{F}]$, then $\mathrm{x}^{\circ}$ is also an $\mathrm{EP}$ for $\mathrm{G}$.

Proof. Suppose, to the contrary, that (1) holds for some $i \varepsilon N$. Then $x^{\circ}$ is not in $X^{1 i}$; since $B_{j(i)}$ is NF, $x^{\circ}$ cannot be $B_{j(i)}$-stable. Thus there exists $y_{j(i)} \varepsilon Y_{j(i)}$ such that changing from $x^{\circ}$ to $\left(x^{\circ}, \mathrm{j}(\mathrm{i}), \mathrm{y}_{j(i)}\right)$ is "advantageous to $B_{j(i)}$ " in the sense defined above. Consider changing the arguments of $F_{j(i)}\left[\left\{f_{k}\left(x^{\circ}\right): k \varepsilon B_{j(i)}\right\}\right]$ one at a time to those of $F_{j(i)}\left[\left\{f_{k}\left(x^{\circ}, j(i), y_{j(i)}\right): k \varepsilon B_{j(i)}\right\}\right]$; by the monotonicity of $F_{j(i)}$ and the transitivity of $\tau_{j(i)}$ it follows-cf. (3)-that $g_{j(i)}\left(x^{\circ}, j(i), y_{j(i)}\right) \tau_{j(i)} g_{j(i)}\left(x^{\circ}\right)$, contradicting the hypothesis that $x^{\circ}$ is an $E P$ for $\left.G B, F\right]$.

We will call a subset $S$ of $N$ unilateral-deviation unanimous (UDU) if, for each $x \varepsilon X$ and $i \varepsilon S$, any $x_{i} \varepsilon X_{i}$ for which $f_{i}\left(x, i, x_{i}\right) f_{i}(x)$ also has the property that

$$
f_{k}\left(x, i, x_{i}\right) Q_{k} f_{k}(x) \text { or } f_{k}\left(x, i, x_{i}\right)=f_{k}(x)
$$

holds for all $k \varepsilon S-\{i\}$. Intuitively, this says that whenever a single player in $S$ has incentive for some particular unilateral deviation from a status quo, then no other player in $\mathrm{S}$ would regret seeing that single deviation effected. This condition, introduced only for the sake of the following Corollary, may be easier to recognize than $\mathrm{NF}$.

CoROLLARY. If each $\mathrm{B}_{j}$ is UDU, and $\mathrm{x}^{\circ}$ is an $\mathrm{EP}$ for $\mathrm{G}[\mathrm{B}, \mathrm{F}]$, then $\mathrm{x}^{\circ}$ is also an $\mathrm{EP}$ for $\mathrm{G}$.

Proof. In view of Theorem 1, it suffices to observe that UDU implies NF; the proof of that implication is straightforward.

As preparation for the next result, observe that condition NF when applied to subset $S$ of $N$ is "local to $S$," in that it does not refer to the attributes $\left\{R_{i}, \varrho_{i}, f_{i}\right\}$ of the players $i \varepsilon N-S$. This is important for ease of checking the condition. Furthermore, $N F$ is "general" in that it does not refer to the attributes $\left(T_{s}, \tau_{s}, F_{s}\right)$ of $S$ as an aggregate player in any particular aggregated version of $G$. The next theorem shows that condition $N F$ is the weakest one, with these two properties, which would suffice for an analog of Theorem 1. 
THEOREM 2. Let all the information needed to define $\mathrm{G}$ and $\mathrm{G}[\mathrm{B}, \mathrm{F}]$ be specified except for $\left(\mathrm{T}_{1}, \tau_{1}, \mathrm{~F}_{1}\right)$ and $\left\{\mathrm{f}_{i}\right.$ : $\left.{ }_{\mathrm{i}} \mathrm{N}-\mathrm{B}_{1}\right\}$. If $\mathrm{B}_{1}$ is not $\mathrm{NF}$, then the missing information can be specified in such a way that $\mathrm{G}[\mathrm{B}, \mathrm{F}]$ has an $\mathrm{EP}$ which is not an $\mathrm{EP}$ for $\mathrm{G}$.

Proof. Since $B_{1}$ is not $N F$, there exists a $B_{1}$-stable $x \varepsilon X$, an $i \varepsilon B_{1}$, and an $x_{i} \varepsilon X_{i}$, such that

$$
f_{i}\left(x, i, x_{i}\right) f_{i}(x)
$$

It follows from (5) that $x$ cannot be an $E P$ for $G$. We shall specify the missing information in such a way that $x$ is an $E P$ for $G[B, F]$.

Choose each of $\left\{f_{t}: t \varepsilon N-B_{1}\right\}$ to be constant, and write " $B(1)$ " instead of " $B_{1}$ " for subscripting purposes. For $x$ to be an $E P$ for $G[B, F]$, it suffices to choose $\left(T_{1}, \tau_{1}, F_{1}\right)$ in such a way that for no $x_{B(1)} \varepsilon X_{B(1)}$ is

$$
F_{1}\left[\left\{f_{p}\left(x, B_{1}, x_{B(1)}\right): p \varepsilon B_{1}\right\}\right] \tau_{1} F_{1}\left[\left\{f_{p}(x): p \varepsilon B_{1}\right\}\right]
$$

Choose $T_{1}=S_{1}$, and $F_{1}$ to be the identity map of $S_{1}$. Define $\tau_{1}^{*}$ as the direct product of $\left\{\varrho_{p}: p \varepsilon B_{1}\right\}$; that is, given $s_{1}$ and $s_{1}^{\prime}$ in $S_{1}$, with respective coordinates $\left\{r_{p}: p \varepsilon B_{1}\right\}$ and $\left\{r_{p}^{\prime}: p \varepsilon B_{1}\right\}$, then $s_{1}^{\prime} \tau_{1}^{*} s_{1}$ holds if and only if

$$
r_{p}^{\prime} \varrho_{p} r_{p} \text { or } r_{p}^{\prime}=r_{p}
$$

holds for all $p \varepsilon B_{1}$, with the former relation holding for at least one $p \varepsilon B_{1}$. This $\tau_{1}^{*}$ is a strict partial order on $T_{1}$, but not in general a linear order. To make $F_{1}$ strictly monotone in each of its arguments, as required, we must (and it suffices to) choose the linear order $\tau_{1}$ to be an extension of $\tau_{1}^{*}$.

The partial order $\tau_{1}^{*}$ can be viewed as a collection of ordered pairs of distinct members of $T_{1}$, where $\left(t, t^{\prime}\right)$ $\varepsilon T_{1} \times T_{1}$ is in the collection if and only if $t \tau_{1}^{*} t^{\prime}$. Now let $t^{\circ}$ be the member of $T_{1}$ with coordinates $\left\{f_{p}(x)\right.$ : $\left.p \varepsilon B_{1}\right\}$, and for each $x_{B(1)} \varepsilon X_{B(1)}$ let $h\left(x_{B(1)}\right)$ be the member of $T_{1}$ with coordinates $\left\{f_{p}\left(x, B_{1}, x_{B(1)}\right): p \varepsilon B_{1}\right\}$. Define a second collection of ordered pairs by

$$
T^{\circ}=\left[h\left(X_{B(1)}\right)-\left\{t^{\circ}\right\}\right] \times\left\{t^{\circ}\right\} .
$$

Then the fact that $x$ is $B_{1}$-stable can be written as $T^{\circ} \cap \tau_{1}^{*}=\phi$, and the desired condition that (6) hold for no $x_{B(1)} \varepsilon X_{B(1)}$ can be written $T^{\circ} \cap \tau_{1}=\phi$.

What remains to be proved, then, is the existence of a strict linear order $\tau_{1}$ on $T_{1}$ which is an extension of $\tau_{1}^{*}$ and which satisfies $T^{\circ} \cap \tau_{1}=\phi$. Since in general $T^{\circ} \neq \phi$, we cannot simply appeal to the general theorem [6] that every partial order can be extended to a linear order. Let $\tau$ be the family of all strict partial orders on $T_{1}$ which contain $\tau_{1}^{*}$ and are disjoint from $T^{\circ} . \tau$ is not empty, since it includes $\tau_{1}^{*}$. Considered as subsets of $T_{1} \times T_{1}$, the members of $\tau$ are partially ordered by set-inclusion, and the union of any linearly-ordered subfamily of $\tau$ is easily shown to be again in $\tau$. By Zorn's Lemma, $\tau$ has a maximal member $\tau_{1}$. The proof will be completed by showing that $\tau_{1}$ is a linear order.

Suppose, to the contrary, that there exist distinct elements $t, t^{\prime}$ of $T_{1}$ such that neither $\left(t, t^{\prime}\right)$ nor $\left(t^{\prime}, t\right)$ lies in $\tau_{1}$. (It is in the balance of this paragraph that a more delicate argument than that given in [6] for the case $T^{\circ}$ $=\phi$ is required.) At most one of $t, t^{\prime}$ can coincide with $t^{\circ}$; if one of them does, choose the notation for $t$ and $t^{\prime}$ so that $t=t^{\circ}$ and $t^{\prime} \neq t^{\circ}$. Observe that at least one of $\left(t, t^{\prime}\right)$ and $\left(t^{\prime}, t\right)$ must fail to be a $\left(t^{\prime \prime}, t^{\prime \prime \prime}\right)$ with the property that $\left(t^{\prime \prime}, t^{\circ}\right) \varepsilon \tau^{\circ}$ and $\left(t^{\prime \prime \prime}, t^{\circ}\right) \varepsilon \tau_{1}$ (this is true because $\left.T^{\circ} \cap \tau_{1}=\phi\right)$; if either $\left(t, t^{\prime}\right)$ or $\left(t^{\prime}, t\right)$ is a $\left(t^{\prime \prime}, t^{\prime \prime \prime}\right)$ with the property just mentioned, choose the notation for $t$ and $t^{\prime}$ so that $\left(t^{\prime}, t\right)$ rather than $\left(t, t^{\prime}\right)$ is such a $\left(t^{\prime \prime}, t^{\prime \prime \prime}\right)$. (This precaution will only be needed if neither $t$ nor $t^{\prime}$ coincides with $t^{\circ}$.) Note for future use that in this case, there cannot be an $s \varepsilon T_{1}$ such that $(s, t) \varepsilon \tau_{1},\left(t^{\prime}, t^{\circ}\right) E \tau_{1}$ and $\left(s, t^{\circ}\right) \varepsilon T^{\circ}$. Observe further that at least one of $\left(t, t^{\prime}\right)$ and $\left(t^{\prime}, t\right)$ must fail to be a $\left(\bar{t}^{\prime \prime}, \bar{t}^{\prime \prime \prime}\right)$ with the property that for some $s \varepsilon T_{1}$, the relations $\left(\mathrm{s}, \bar{t}^{\prime \prime}\right) \varepsilon T_{1},\left(\bar{t}^{\prime \prime \prime}, t^{\circ}\right) \varepsilon \tau_{1}$ and $\left(s, t^{\circ}\right) \varepsilon T^{\circ}$ all hold. In this case, choose the notation for $t$ and $t^{\prime}$ so that $\left(t, t^{\prime}\right)$ is not a $\left(\vec{t}^{\prime \prime}, t^{\prime \prime \prime}\right)$ with the last-mentioned property. (This precaution will only be needed if neither $t$ nor $t^{\prime}$ coincides with $t^{\circ}$, and if in addition neither $\left(t, t^{\prime}\right)$ nor $\left(t^{\prime}, t\right)$ is a $\left(t^{\prime \prime}, t^{\prime \prime \prime}\right)$ of the type described above.) 


$$
\begin{gathered}
\bar{\tau}_{1}=\tau_{1} \cup\left\{\left(t, t^{\prime}\right)\right\} \cup\left\{\left(s, t^{\prime}\right):(s, t) \varepsilon \tau_{1}\right\} \cup\left\{\left(t, s^{\prime}\right):\left(t^{\prime}, s^{\prime}\right) \varepsilon \tau_{1}\right\} \\
\cup\left\{\left(s, s^{\prime}\right):(s, t),\left(t^{\prime}, s^{\prime}\right) \varepsilon \tau_{1}\right\} .
\end{gathered}
$$

Then $\bar{\tau}_{1}$ is a binary relation on $T_{1}$ which is a proper extension of $\tau_{1}$. A straightforward enumeration of cases, ignoring the care with which the notation for $t$ and $t^{\prime}$ was chosen above, verifies that $\bar{\tau}_{1}$ is a strict partial order on $T_{1}$. With that care taken into account, another enumeration of cases verifies that $T^{\circ} \cap \bar{\tau}_{1}=\phi$, yielding a contradiction to the maximality of $\tau_{1}$ in $\tau$. So $\tau_{1}$ is a linear order, and Theorem 2 is proved.

Having considered what conditions rule out the introduction through aggregation of "extraneous" solutions ( $E P$ 's), we now turn to ruling out the loss of solutions under aggregation. A subset $S$ of $N$ will be called unilateral-deviation strong (UDS) if for each $x \varepsilon X$, in case $f_{k}\left(x, S, x_{s}\right) \varrho_{k} f_{k}(x)$ holds for any $k \varepsilon S$ and $x_{s} \varepsilon X_{s}$, it follows that $f_{i}\left(x, i, x_{i}\right) \varrho f_{i}(x)$ for some $i \varepsilon S$ and $x_{i} \varepsilon X_{i}$. This (somewhat unsatisfactory) terminology is intended to reflect the idea that the unilateral deviations desirable to the individual members of $S$ (as deviators) are, taken together, sufficiently "strong" to reject any status quo $x$ from which $S$ as a collective could possibly deviate to the advantage of any of its members.

THEOREM 3. If each $\mathrm{B}_{j}$ is UDS, and $\mathrm{x}^{\circ}$ is an $\mathrm{EP}$ for $\mathrm{G}$, then $\mathrm{x}^{\circ}$ is also an $\mathrm{EP}$ for $\mathrm{G}[\mathrm{B}, \mathrm{F}]$.

Proof. Suppose, to the contrary, that $g_{j}\left(x^{\circ}, j, y_{j}\right) \tau_{j} g_{j}\left(x^{\circ}\right)$ for some $j \varepsilon M$ and some $y_{j} \varepsilon Y_{j}$. Because each of $\left\{\varrho_{k}\right.$ : $\left.k \varepsilon B_{j}\right\}$ is a linear order and $F_{j}$ is monotone, it follows (proof by contradiction) that

$$
f_{k}\left(x^{\circ}, j, y_{j}\right) \varrho_{k} f_{k}\left(x^{\circ}\right)
$$

must hold for at least one $k \varepsilon B_{j}$. From this, and the fact that $B_{j}$ is $U D S$, there follows immediately a contradiction to the hypothesis that $x^{\circ}$ is an $E P$ of $G$.

Note that $U D S$, applied to subset $S$ of $N$, has the same "local to S" and "generality" properties described before Theorem 2. The next theorem shows that UDS is the weakest condition, with these two properties, which would suffice for an analog of Theorem 3 .

THEOREM 4. Let all the information needed to define $\mathrm{G}$ and $\mathrm{G}[\mathrm{B}, \mathrm{F}]$ be specified except for $\left(\mathrm{T}_{1}, \tau_{1}, \mathrm{~F}_{1}\right)$ and $\left\{\mathrm{f}_{i}\right.$ : ie $\left.\mathrm{N}-\mathrm{B}_{1}\right\}$. If $\mathrm{B}_{\mathrm{1}}$ is not UDS, then the missing information can be specified in such a way that $\mathrm{G}$ has an $\mathrm{EP}$ which is not an EP for $\mathrm{G}[\mathrm{B}, \mathrm{F}]$.

Proof. Write " $B(1)$ " instead of " $B_{1}$ " for subscripting purposes. Since $B_{1}$ is not $U D S$, there exists an $x \varepsilon X$, an $x_{B(1)} \varepsilon X_{B(1)}$, and a $k \varepsilon B_{1}$ such that

$$
\begin{gathered}
f_{k}\left(x, B_{1}, x_{B(1)}\right) \varrho_{k} f_{k}(x), \\
f_{i}\left(x, i, x_{i}\right) \varrho_{i} f_{i}(x) \text { for no } i \varepsilon B_{1} \text { and } x_{i} \varepsilon X_{i} .
\end{gathered}
$$

Choose each of $\left\{f_{t}: t \varepsilon N-B_{1}\right\}$ to be constant; then (8) implies that $x$ is an $E P$ for $G$. Choose $T_{1}=S_{1}$, and $F_{1}$ to be the identity map of $S_{1}$. Let $\left|B_{1}\right|=b$, and let $\{k(1), k(2), \ldots, k(b)\}$ be any enumeration of $B_{1}$ in which $k(1)$ $=k$. Take $\tau_{1}$ to be the lexicographic product of the sequence $\left\{\varrho_{k}=\varrho_{k(1)}, \varrho_{k(2)}, \ldots, \varrho_{k(b)}\right\}$; then $\tau_{1}$ is a linear order and $F_{1}$ is monotone. It follows from (7) that $g_{1}\left(x, B_{1}, x_{B(1)}\right) \tau_{1} g_{1}(x)$, and so $x$ cannot be an $E P$ for $\left.G B, F\right]$, completing the proof.

Combining the previous results, we see that if each $B_{j}$ is both $N F$ and $U D S$, then $\left.G B, F\right]$ has the same set of $E P$ 's as $G$. Furthermore, the conjunction " $N F$ and $U D S$ " is the weakest condition, with the properties of being "local" and "general" as defined earlier, which suffices for such a conclusion; one might well refer to this condition as "aggregability." The question of how this condition might be systematically and efficiently checked-for is a natural one, but will not be addressed here. 
To relate the preceding material to that of [1], we define a subset $S$ of $N$ to be limited-dependent $(L D)$ if, for each i $\mathrm{E}, f_{i}(x)$ does not depend on the coordinates of $x$ associated with the players in $S-\{i\}$. Theorems 1 and 2 of [1] are then equivalent to the following assertion.

THEOREM 5. If each $\mathrm{B}_{j}$ is $\mathrm{LD}$, then $\mathrm{G}[\mathrm{B}, \mathrm{F}]$ has the same set of $\mathrm{EP}$ 's as $\mathrm{G}$.

Proof. By Theorems 1 and 3, it suffices to show, if $S$ is an $L D$ subset of the players, that $S$ is both $U D U$

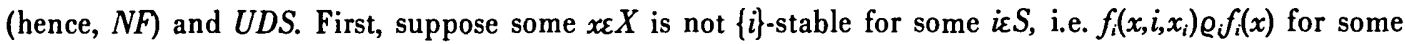
$x_{i} \varepsilon X_{i}$. Since $S$ is $L D, f_{k}\left(x, i, x_{i}\right)=f_{k}(x)$ for all $k \varepsilon S-\{i\}$. This shows that $S$ is $U D U$. Next, consider any $x \varepsilon X$ such that $f_{k}\left(x, S, x_{S}\right) \varrho_{k} f_{k}(x)$ for some $k \varepsilon S$ and $x_{s} X_{s}$. Let $x_{k}$ be the coordinate of $x_{s}$ corresponding to player $k$; since $S$ is $L D, f_{k}\left(x, S, x_{S}\right)=f_{k}\left(x, k, x_{k}\right)$ and so $f_{k}\left(x, k, x_{k}\right) \varrho_{k} f_{k}(x)$. This shows that $S$ is $U D S$, completing the proof of the theorem.

To show that Theorem 5's sufficient condition for preservation of the solution-set was not also a necessary condition, the following example was given in [1]. It had $n=2, m=1$, and $B_{1}=\{1,2\}$. Each $\varrho_{i}(i=1,2)$ was the numerical " $>$ " relation, $X_{1}=\{A, B\}, X_{2}=\{a, b\}$, and the payoff functions $f_{1}$ and $f_{2}$ were identical $\left(f_{1}=f_{2}=\bar{f}\right)$ with

$$
\bar{f}(A, a)=2, \bar{f}(A, b)=\bar{f}(B, a)=1, \bar{f}(B, b)=0 .
$$

Here $B_{1}$ is not $L D$, but both $G$ and $\left.G B, F\right]$-for any choice of $\left(T_{1}, \tau_{1}, F_{1}\right)$-have $(A, a)$ as the unique equilibrium point. It is easily verified that $B_{1}$ is both $N F$ and $U D S$, so that this example is "explained" by Theorems 1 and 3 of the present paper.

To illustrate that the hypotheses of Theorems 1 and 3, though sufficient conditions for the Theorems' conclusions to hold, are not also necessary conditions, we will give an example in which $N F$ and $U D S$ both fail, but $G$ and $G B, F]$ have the same set of equilibrium points. We would like the set of $G$ 's equilibrium points to be a proper subset of $X$ (so that aggregation has a fair chance to introduce one or more extraneous $E P$ 's), and to be nonempty (so that aggregation has a fair chance to lose one or more $E P$ 's). Also, to keep the example simple, we would like to have $n=2, m=1$, and $B_{1}=\{1,2\}=N$. For an example meeting all these stipulations, take $\left(X_{1}, X_{2}, \varrho_{1}, \varrho_{2}\right)$ as in the last paragraph, but set

$$
f_{1}(A, a)=1, f_{1}(B, a)=2, f_{1}(A, b)=f_{1}(B, b)=0
$$

and $f_{2}=-f_{1}$. Then (in the notation introduced above eq (4)) $X^{11\}}=X-\{(A, a)\}$ and $X^{\mid 2\}}=\{(A, b),(B, b)\}$. Because $G$ is zero-sum, $X^{1,2\}}$ is all of $X$, and comparison with (4) shows that $N F$ fails. That $U D S$ fails can be seen from the statement of this condition by taking $k=1, x=(A, b), x_{s}=(B, a)$. The set of equilibrium points of $G$ is given by $X^{11\}} \cap X^{\{2\}}=X^{\{2\}}$, a nonempty proper subset of $X$. Now take $T_{1}=S_{1}, F_{1}$ to be the identity map of $T_{1}$, and $\tau_{1}$ to be the lexicographic product of the ordered pair $\left(\varrho_{2}, \varrho_{1}\right)$; with this choice, $G[B, F]$ has the same equilibrium-point set as $G$.

In the remainder of this Section, we investigate how well the conditions appearing above-NF, $U D U$, $U D S$, "NF and UDS," and $L D$-conform to some intuitively plausible requirements for representing the notion of "parallel interests." Let $\exists$ be a family of nonempty subsets of $N$, which includes all singletons. We will say that 7 is equivalence-derived if there is an equivalence relation on $N$ such that subset $S$ of $N$ belongs to $\mathcal{Y}$ if and only if $S$ lies in a single equivalence class. Also, we will call $\mathcal{Y}$ hereditary if $\varnothing \neq T \subset S$ and $S \varepsilon \neg$

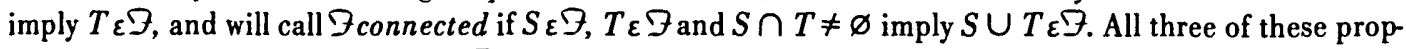
erties are plausible requirements if $\mathcal{Y}$ is to represent the family of all subsets whose players "have parallel interests." It is easy to show that a family $\mathcal{Y}$ is equivalence-derived if and only if it is both hereditary and connected, so that we will deal with these last two properties.

None of the conditions appearing in the previous theorems necessarily yields a connected family of subsets of $N$. To show this, it suffices to give an example in which subsets $S$ and $T$ of the players are both $L D$ (hence $N F, U D U$ and $U D S$ ), satisfy $S \cap T \neq \varnothing$, but have $S \cup T$ neither $N F$ nor $U D S$. Such an example can be constructed from the preceding one by adjoining a third player as "dummy." Specifically, take $\left(X_{1}, X_{2}, \varrho_{1}, \varrho_{2}\right)$ as before; $\left(X_{3}, \varrho_{3}\right)$ need not be specified, for what follows. Adjoin a dummy argument $x_{3} \varepsilon X_{3}$ to 
the previous definitions of $f_{1}$ and $f_{2}$, and take $f_{3}$ to be constant. Choosing $S=\{1,3\}$ and $T=\{2,3\}$ then yields an example with the desired characteristics.

It is easily shown that the $L D$ subsets of $N$ form a hereditary family, as do the $U D U$ subsets. To dispose of the "hereditary question" for the remaining properties, it suffices to give an example in which subsets $S$ and $T$ of the players satisfy $\varnothing \neq T \subset S, S$ is both $N F$ and $U D S$, but $T$ is neither $N F$ nor $U D S$. This example, too, will be constructed from a preceding one by suitably adjoining a third player. Take $\left(X_{1}, X_{2}, \varrho_{1}, \varrho_{2}\right)$ as before, and take $X_{3}=\{\alpha, \beta\} ; \varrho_{3}$ is the numerical " $>$ " relation. Following the model of the previous example, let

$$
f_{1}(A, a, \alpha)=1, f_{1}(B, a, \alpha)=2, f_{1}(A, b, \alpha)=f_{1}(B, b, \alpha)=0
$$

and $f_{2}\left(x_{1}, x_{2}, \alpha\right)=-f_{1}\left(x_{1}, x_{2}, \alpha\right)$ for all $\left(x_{1}, x_{2}\right) \varepsilon X_{1} \times X_{2}$. This is already enough to assure that $T=\{1,2\}$ is neither $N F$ nor $U D S$. To make $S=\{1,2,3\}$ both $N F$ and $U D S$, set

$$
\begin{gathered}
f_{1}\left(x_{1}, x_{2}, \beta\right)=3 \text { for all }\left(x_{1}, x_{2}\right) \in X_{1} \times X_{2}, \\
f_{2}\left(x_{1}, a, \beta\right)=3, f_{2}\left(x_{1}, b, \beta\right)=4 \text { for all } x_{1} \varepsilon X_{1}, \\
f_{3}\left(x_{1}, x_{2}, \alpha\right)=0 \text { for all }\left(x_{1}, x_{2}\right) \varepsilon X_{1} \times X_{2}, \\
f_{3}\left(x_{1}, a, \beta\right)=0, f_{3}\left(x_{1}, b, \beta\right)=1 \text { for all } x_{1} \varepsilon X_{1} .
\end{gathered}
$$

An initial impression from these findings might be, that despite the motivating observation with which the present paper began, aggregability of a subset of the players does not really have much to do with the parallelism of those players' interests. I presently prefer an alternative interpretation, namely that the findings are corrective to an implicit assumption that parallelism of players' interests must (like parallelism of sets of lines in the Euclidean plane) be viewed as equivalence-derived from a binary relation of "parallel interests" between individual pairs of players. The formulation of a different concept of parallel interests for a subset of the players, and the study of the relationship between that concept and aggregability, are planned for a subsequent paper.

\section{Approximations}

Since the topics of this section deal with quantitative rather than qualitative relationships, we now take all sets $R_{i}$ and $T_{j}$ to be the set of real numbers, and take all the relations $\varrho_{i}$ and $\tau_{j}$ to be the ordinary numerical "greater than" relation. Theorems 1 and 3 involve the notions of an equilibrium point, of an aggregation of a given game $G$, and of conditions $N F$ and $U D S$. The definitions of these concepts involve elements of an essentially order-theoretic nature (the linear-order properties of $\varrho_{i}$ and $\tau_{j}$; the monotonicity of $F_{j}$ ). To obtain "approximate" versions of the theorems, it will be necessary to replace these "ordinal" elements by suitable "cardinal" ones. The particular replacements introduced below appear reasonable, but other plausible alternatives may be more appropriate in particular contexts.

For each is $\mathrm{N}$ and each $x \varepsilon \mathrm{X}$, the quantity

$$
M_{i}(x)=\sup \left\{f_{i}\left(x, i, x_{i}\right): x_{i} \varepsilon X_{i}\right\}-f_{i}(x)
$$

is nonnegative. If $\delta=\left(\delta_{1}, \ldots, \delta_{n}\right)$ is an $n$-tuple of positive real numbers, and if $x^{\circ} \varepsilon X$ satisfies

$$
M_{i}\left(x^{\circ}\right) \leqslant \delta_{i}(\text { all } i \varepsilon N)
$$

then $x^{\circ}$ will be called a $\delta-E P$ of game $G$. Approximate $E P$ 's of $\left.G B, F\right]$ are defined similarly. 
We will say that $F_{j}$ is $\left(k_{j}^{+}, k_{j}^{-}\right)$-bounded monotone in each argument, where $k_{j}^{+}$and $k_{j}^{-}$are positive constants, if for each $s_{j} \varepsilon S_{j}$, and for any $i \varepsilon B_{j}$ with $r_{i}$ the $i$-th coordinate of $s_{j}$, and for any $r_{i}^{\prime} \varepsilon R_{i}$,

$$
r_{i}^{\prime}>r_{i} \text { implies } 0<k_{j}^{-} \leqslant\left[F_{j}\left(s_{j}, i, r_{i}^{\prime}\right)-F_{j}\left(s_{j}\right)\right] /\left(r_{i}^{\prime}-r_{i}\right) \leqslant k_{j}^{+} .
$$

This hypothesis expresses the plausible idea that an aggregate player's payoff should respond "in a bounded manner" to changes in the payoffs of the individual players comprising the aggregate. Note that (10) holds with $k_{j}^{+}=k_{j}^{-}=1$ if $F_{j}\left(s_{j}\right)$ is the sum (as in [5] of the coordinates of $s_{j}$.

For a given $c>0$, a subset $S$ of $N$ will be called $N F(c)$ if for all $x \varepsilon X$, if there is an $i \varepsilon S$ and an $x_{i} \varepsilon X_{i}$ such that $f_{i}\left(x, i, x_{i}\right)>f_{i}(x)$, then there is an $x_{s} X_{s}$ such that $f_{k}\left(x, S, x_{s}\right) \geqslant f_{k}(x)$ for all $k \varepsilon S$ and

$$
f_{k}\left(x, S, x_{s}\right)-f_{k}(x) \geqslant c\left[f_{i}\left(x, i, x_{i}\right)-f_{i}(x)\right]
$$

holds for at least one $k \varepsilon S$. (The intuitive interpretation of this condition follows readily from that given earlier for $N F$.)

THEOREM 6. Assume $\mathrm{F}_{j}$ is $\left(\mathrm{k}_{j}^{+}, \mathrm{k}_{j}^{-}\right)$-bounded monotone and $\mathrm{B}_{j}$ is $\mathrm{NF}\left(\mathrm{c}_{j}\right)$ for all jeM. Let $\mathrm{d}$ be a positive $\mathrm{n}$-tuple and $\delta^{\prime}$ a positive m-tuple such that $\delta_{i} \geqslant \delta_{j(i)}^{\prime} / \mathrm{k}_{j(i)}^{-} \mathrm{c}_{j(i)}$ for all $\mathrm{i \varepsilon N}$. If $\mathrm{x}^{\circ}$ is a $\delta^{\prime}-\mathrm{EP}$ for $\mathrm{G}[\mathrm{B}, \mathrm{F}]$, then $\mathrm{x}^{\circ}$ is also a d-EP for $\mathrm{G}$.

Proor. Suppose, to the contrary, that $f_{i}\left(x^{\circ}, i, x_{i}\right)-f_{i}\left(x^{\circ}\right)>\delta_{i}$ for some $i \varepsilon S$ and some $x_{i} \varepsilon X_{i}$. Let $j=j(i)$. Since $B_{j}$ is $N F\left(c_{j}\right)$, there is a $y_{j} \varepsilon Y_{j}$ such that $f_{k}\left(x^{\circ}, j, y_{j}\right) \geqslant f_{k}\left(x^{\circ}\right)$ for all $k \varepsilon B_{j}$, with

$$
f_{k}\left(x^{\circ}, j, y_{j}\right)-f_{k}\left(x^{\circ}\right) \geqslant c_{j}\left[f_{i}\left(x^{\circ}, i, x_{i}\right)-f_{i}\left(x^{\circ}\right)\right]>c_{j} \delta_{i}
$$

holding for at least one $k_{\varepsilon} B_{j}$. Consider changing the arguments of $F_{j}\left[\left\{f_{k}\left(x^{\circ}\right): k \varepsilon B_{j}\right\}\right]$ one at a time to those of $F_{j}\left[\left\{f_{k}\left(x^{\circ}, j, y_{j}\right): k \varepsilon B_{j}\right\}\right]$, beginning with a $k \varepsilon B_{j}$ for which (12) holds. It follows from (10) and the last display that

$$
g_{j}\left(x^{\circ}, j, y_{j}\right)-g_{j}\left(x^{\circ}\right)>k_{j}^{-} c_{j} \delta_{i}
$$

yielding a contradiction to the assumption that $x^{\circ}$ is a $\delta^{\prime}-\mathrm{EP}$ for $\mathrm{G}[\mathrm{B}, \mathrm{F}]$.

Theorem 6 is an "approximate" version of Theorem 1. To obtain an "approximate" version of Theorem 3 , we first define subset $S$ of $N$ to be $U D S(c)$, where $c>0$, if for all $x \varepsilon X$, if $f_{k}\left(x, S, x_{S}\right)>f_{k}(x)$ holds for some $k \varepsilon S$ and $x_{s} \varepsilon X_{s}$, then

$$
f_{i}\left(x, i, x_{i}\right)-f_{i}(x) \geqslant c\left[f_{k}\left(x, S, x_{S}\right)-f_{k}(x)\right]
$$

holds for some $i \varepsilon S$ and $x_{i} \varepsilon X_{i}$. (The intuitive interpretation follows from that given earlier for UDS.)

THEOREM 7. Assume $\mathrm{F}_{j}$ is $\left(\mathrm{k}_{j}^{+}, \mathrm{k}_{j}^{-}\right)$-bounded monotone and $\mathrm{B}_{j}$ is UDS $\left(\mathrm{c}_{j}\right)$ for all jeM. Let $\mathrm{d}$ be a positive $\mathrm{n}-$ tuple and $\delta^{\prime}$ a positive m-tuple such that $\delta_{i} \leqslant c_{j(i)} \delta^{\prime}{ }_{j(i)} / \mathrm{k}_{j(i)}^{+}\left|\mathrm{B}_{j(i)}\right|$ for all $\mathrm{i} \mathrm{N}$. If $\mathrm{x}^{\circ}$ is a $\delta \cdot \mathrm{EP}$ for $\mathrm{G}$, then $\mathrm{x}^{\circ}$ is also a d'-EP for $\mathrm{G}[\mathrm{B}, \mathrm{F}]$.

PRoof. Suppose, to the contrary, that $g_{j}\left(x^{\circ}, j, y_{j}\right)-g_{j}\left(x^{\circ}\right)>\delta_{j}^{\prime}$ for some $j \varepsilon M$ and $y_{j} \varepsilon Y_{j}$. Consider changing the arguments of $F_{j}\left[\left\{f_{k}\left(x^{\circ}\right): k \varepsilon B_{j}\right\}\right]$ one at a time to those of $F_{j}\left[\left\{f_{k}\left(x^{\circ}, j, y_{j}\right): k \varepsilon B_{j}\right\}\right]$; it follows from (10) that

$$
\begin{aligned}
g_{j}\left(x^{\circ}, j, y_{j}\right)-g_{j}\left(x^{\circ}\right) & \leqslant k_{j}^{+} \Sigma\left\{\max \left[0 f_{k}\left(x^{\circ}, j, y_{j}\right)-f_{k}\left(x^{\circ}\right)\right]: k \varepsilon B_{j}\right\} \\
& \leqslant k_{j}^{+}\left|B_{j}\right| \max \left\{f_{k}\left(x^{\circ}, j, y_{j}\right)-f_{k}\left(x^{\circ}\right): k \varepsilon B_{j}\right\}
\end{aligned}
$$


Thus there must be a $k \varepsilon B_{j}$ for which

$$
f_{k}\left(x^{\circ}, j, y_{j}\right)-f_{k}\left(x^{\circ}\right)>\delta_{j}^{\prime} / k_{j}^{\dagger}\left|B_{j}\right|
$$

Since $B_{j}$ is $U D S\left(c_{j}\right)$, it follows that there must be an $i \varepsilon B_{j}$ and an $x_{i} \varepsilon X_{i}$ such that

$$
f_{i}\left(x^{\circ}, i, x_{i}\right)-f\left(x^{\circ}\right)>c_{j} \delta_{j}^{\prime} / k_{j}^{+}\left|B_{j}\right|
$$

yielding a contradiction to the assumption that $x^{\circ}$ is a $\delta-E P$ for $G$.

Note that the presence of $k_{j}^{+}$in (10) is required for Theorem 7 but not for Theorem 6, and vice versa for $k_{j}^{-}$ (so long as $F_{j}$ is assumed monotone). The two theorems are not intended to apply simultaneously to the same pair $\left(\delta, \delta^{\prime}\right)$. Theorem 6 directly generalizes Theorem 3 of [1], an extension of which is obtained by taking all $c_{j}=1$, all $k_{j}^{+}=k_{j}^{-}=1$, and each $F_{j}\left(s_{j}\right)$ to be the sum of the coordinates of $s_{j}$. Making the same choices in Theorem 7 yields a result closely related to Theorem 4 of [1].

\section{References}

[1] Goldman, A. J.; Shier, D. R. Player aggregation in noncooperative games. J. Res. Nat. Bur. Stand (U.S.). 85(5): 391-396; 1980 September-0ctober.

[2] Goldman, A. J.; Pearl, M. H. The dependence of inspection-system performance on levels of penalties and inspection resources. J. Res. Nat. Bur. Stand. (U.S.). 80B(2): 189-236; 1976 April-June.

[3] Pearl, M. H.; Goldman, A. J. Policing the marketplace. Mathematics Magazine. 50(4): 179-185; 1977 September.

[4] Pearl, M. H.; Goldman, A. J. A game-theoretic model of inspection-resource allocation. J. Res. Nat. Bur. Stand. (U.S.). 86(2): 193-214; 1981 March-April.

[5] Szidarovszky, F.; Galántai, A. On new concepts of game theory, in Applied Numerical Modeling, C. A. Brebbia, ed. New York, NY: Halsted Press, John Wiley \& Sons; 1978. 587-593.

[6] Szpilrajn, E. Sur l'Extension de l'Ordre Partiel. Fund. Math. 16: 386-389; 1930. 